\title{
Melatonin and Testicular Damage in Busulfan Treated Mice
}

\author{
Mehri Mirhoseini ${ }^{1}$; Ghasem Saki $^{2, *}$; Masoud Hemadi ${ }^{3}$; Ali Khodadadi ${ }^{4}$; Javad Mohammadi \\ Asl $^{4}$ \\ ${ }^{1}$ Department of Anatomy, School of Medicine, Ahvaz Jundishapur University of Medical Sciences, Ahvaz, IR Iran \\ ${ }_{2}^{2}$ Physiology Research Center, School of Medicine, Ahvaz Jundishapur University of Medical Sciences, Ahvaz, IR Iran \\ ${ }_{3}$ Fertility, Infertility and Perinatology Research Center, School of Medicine, Ahvaz Jundishapur University of Medical Sciences, Ahvaz, IR Iran \\ ${ }^{4}$ Cancer, Petroleum and Environmental Pollutants Research Center, Ahvaz Jundishapur University of Medical Sciences, Ahvaz, IR Iran \\ ${ }^{*}$ Corresponding Author: Ghasem Saki, Physiology Research Center, School of Medicine, Ahvaz Jundishapur University of Medical Sciences, Ahvaz, IR Iran. Tel: +98-9166181685, Fax: \\ +98-6113335399, E-mail: ghasemsaki@yahoo.com
}

Received: August 27, 2013; Revised: October 5, 2013; Accepted: October 26, 2013

\begin{abstract}
Background: Advancement in the treatment of various types of cancer has led to greater patient survival. These treatments essentially have toxic effects on different kinds of cells, such as germ cells. Infertility as one of the side effects of cancer treatment has changed the quality of life of young cancer survivors dramatically. Melatonin is an antioxidant with receptors in the reproductive systems.

Objectives: We supposed that melatonin, as an antioxidant, may protect testis against the toxic effects of the drugs.

Materials and Methods: In this experimental study, three groups with seven mice each, were allocated. The control group received normal saline for two months, and the busulfan group received a single dose of $40 \mathrm{mg} / \mathrm{kg}$ busulfan intra-peritoneally, and the melatonin group received $20 \mathrm{mg} / \mathrm{kg}$ melatonin daily for two months, 45 days after a single dose of busulfan. Next, after decapitation and removal of the testis, tissues were fixed in Bouin's solution and stained by H\&E and TUNEL. The sections were evaluated, assessing morphology and spermatogenesis.

Results: In this research, a significant reduction in Johnson's criteria in the busulfan group (Mean rank $=15.50$ ) was found versus the control group (Mean rank =45.50), $\mathrm{P}<0.001$ and in the melatonin group (Mean rank=45.50) compared to the busulfan group (Mean rank $=15.50), \mathrm{P}<0.001$. There was a significant difference between the melatonin and control groups, $\mathrm{P}<0.05$. In addition, a significant decrease in seminiferous tubule diameter was observed in the busulfan group (763.2 \pm 104.41$)$ versus the control group $(855.4 \pm 52.35)$, $\mathrm{P}<0.01$ and melatonin group (834.2 \pm 87.26$), \mathrm{P}<0.05$. Testicular epithelium height was significantly decreased in the busulfan group (Mean rank $=$ 14.60) compared to the control group (Mean rank $=26.40$ ), $\mathrm{P}<0.01$ and in the busulfan group (Mean rank=14.95) in comparison with the melatonin group (Mean rank = 26.05), $\mathrm{P}<0.01$. Also melatonin group (Mean rank=25.42) showed a significant reduction in epithelium height compared to the control group (Mean rank $=35.58$ ), $\mathrm{P}<0.05$. Spermatogenesis was impaired in the busulfan group. Although melatonin reduced the rate of apoptosis in the busulfan group, yet it could not remove all apoptotic cells.

Conclusions: This study indicated that melatonin ameliorates the cytotoxic effects of busulfan on germ cells.
\end{abstract}

Keywords: Melatonin; Spermatogenesis; Busulfan; Mice; Testis

\section{Background}

An extensive development in childhood cancer treatment in the recent years has led to a growing population of survivors of childhood malignancies. Therefore, life expectancy has increased (1). Cancer treatments are highly effective, but they cannot specifically target tumoral cells. A major problem in this regard is infertility due to oncological treatments (2). Alkylating agents, which are a kind of cytotoxic chemotherapy drug, can damage the germinal epithelium leading to oligospermia or azoospermia (2). Many attempts have been made to treat infertility of cancer survivors so far. Although semen freezing is a common clinical practice in adults, and fertility rate especially in intra-cytoplasmic sperm injection (ICSI) has greatly improved, this method cannot be applied for young men (3). In fact, there are only Sertoli cells and different types of spermatogonia in immature testicular tissue, which the stem cells are found among them (4).

Busulfan (1, 4-butanediol dimethanesulfonate [myleran]) is a bifunctional alkylating agent and is used for the treatment of various malignant diseases, such as chronic myelogenous leukemia and polycythemia vera $(4,5)$. Busulfan is a highly toxic agent, which can induce various adverse effects, both acute and chronic, in several biological organs such as hematologic (6), nervous (7) and reproductive organs (8). Using alkylating agents in chemotherapy may induce various cellular mechanisms in germ

Implication for health policy/practice/research/medical education:

Infertility as one of the side effects of cancer treatment has changed the quality of life of young cancer survivors dramatically. This research indicates that antioxidants such as melatonin treatment after chemotherapy in patients with cancer may ameliorate drug side effects. It may also be used for the protection of germinal layer defects due to radiotherapy or chemotherapy in human.

Copyright (C) 2014, Iranian Red Crescent Medical Journal; Published by Kowsar Corp. This is an open-access article distributed under the terms of the Creative Commons Attribution License, which permits unrestricted use, distribution, and reproduction in any medium, provided the original work is properly cited. 
cells and somatic cells. Transferring the alkyl group(s) to various cellular constituents can cause cytotoxic effects. However, DNA alkylation events may constitute major incitements leading to cell death (9). Simone et al. declared that busulfan kills cells by producing free radicals (10). Antioxidants neutralize both free radicals and the oxidative reactions caused by them (10). Melatonin (MLT), an endogenous indolamine is produced in mammalian pineal glands. Researches during 1993 demonstrated that melatonin functions as a direct free radical scavenger, which can detoxify highly reactive hydroxyl free radicals $(\mathrm{OH})$ in vitro (11). Moreover, numerous publications have approved that melatonin and its metabolites can reduce oxidative stress in vitro (11). Nowadays researchers believe that melatonin receptors exist in many areas of the brain, the pituitary gland and peripheral tissues such as the reproductive organs $(12,13)$. So far, several studies have been performed on the effects of melatonin on the reproductive system (14-17). However, reports have shown rather contradictory results of antioxidant activities in male germ cells.

\section{Objectives}

The aim of the present research was to examine melatonin's ability to improve the quality of spermatogenesis.

\section{Materials and Methods}

\subsection{Animals}

In this study, twenty-one male NMRI (Naval Medical Research Institute) mice ( 6 - 7 weeks old, 25 - 30 g) were used. The experimental animals were obtained from Ahvaz Jundishapur University of Medical Sciences, Experimental animal Research Center. This study was approved by the ethics committee of Jundishapur University (code; Ajums.rec.1392.125 date; 2013.10.5), and performed according to provided guidelines. Standard laboratory conditions were kept (12 hours dark and 12 hours light cycle, relative humidity of $50 \pm 5 \%$ and temperature of $22 \pm 3^{\circ} \mathrm{C}$ ) one week before the experiment and those conditions were preserved until the end of the experiment. Animal cages were kept clean, and commercial food (pellet) and water were provided ad libitum.

\subsection{Grouping}

Sample size was calculated as two mice per group by the following formula: $\mathrm{N}=$ with a power of 0.9 and type II error of 0.1; however, we assigned 7 mice to each group. The test power was 0.896. Mice in Busulfan group received a single dose of $40 \mathrm{mg} / \mathrm{kg}$ busulfan intra peritoneally and melatonin group received $20 \mathrm{mg} / \mathrm{kg}$ melatonin daily for two months, 45 days after busulfan treatment. In the control group, mice received intra-peritoneal injection of normal saline daily for two months. Busulfan dose was assigned based on previous researches that demonstrated the toxic effect of busulfan on testes (16), and melatonin dose was selected based on previous reports demonstrating its anti-oxidative effect (14).

\subsection{Organ Removal and Tissue Processing}

Animals were killed by decapitation under ether anesthesia, and testes of the animals were removed and fixed in Bouin's solution. The samples were dehydrated and paraffin block were prepared. Next, $5 \mu \mathrm{m}$ serial sections were prepared, and for histological assessment five slides from each testis were stained with hematoxylin and eosin and TUNEL staining for apoptosis assessment. Samples were analyzed under blindfold conditions.

\subsection{Histopathology}

The seminiferous tubules diameters and the epithelium thicknesses were evaluated by using calibrated linear scale of the Analysis LS Starter software in the $10 \mathrm{X}$ eyepiece of Olympus microscope at 40X objective lens. Only circular and near circular tubules were examined. The seminiferous epithelium thickness was calculated by subtracting the lumen diameter from the tubule diameter divided by two $(18,19)$.

\subsection{Assessment of Spermatogenesis}

Light microscopy was used for the evaluations. Testicular damage and spermatogenesis were graded histopathologically using the Johnson's mean testicular biopsy score (MTBS criteria) (20). Johnson scoring is a common technique to evaluate spermatogenesis. By using a $40 \times$ magnification, 100 tubules for each animal were assessed and each tubule was given a grade ranging from 1 to 10 . Score one was considered for the tubules with complete inactivity, and score 10 for those with maximum activity (at least five or more spermatozoa in the lumen).

\subsection{TUNEL Assay (TdT-Mediated dUTP-X Nicked end Labeling)}

TUNEL test was performed according to the manufacturer's instructions. After fixation in Bouin's solution, tissues were dehydrated in ethanol (alternatively 70\%, 90\%, and $100 \%$ ) and embedded in paraffin. Before the TUNEL staining, testicular sections $(5 \mu \mathrm{m})$ were rehydrated (xylene 5 minutes; ethanol 100\%, 95\%, 70\%, 2 minutes each) and washed in distilled water. The sections were incubated with proteinase $\mathrm{K}(20 \mu \mathrm{g} / \mathrm{mL}$ in $10 \mathrm{mM}$ Tris/HCL, PH 7.48) at room temperature for 30 minutes and washed with PBS. Sections were re-washed two times with PBS and incubated in a moist chamber with the TUNEL reaction mixture (calf thymus terminal deoxy nucleotidyl transferase, recombinant in E. coli, in storage buffer) for 60 minutes at $37^{\circ} \mathrm{C}$. The sections were incubated in POD after washing two times with PBS (anti fluorescein antibody, FAB 
fragment from sheep, conjugated with horse-radish peroxidase) in a moist chamber for 10 minutes at $37^{\circ} \mathrm{C}$. After three PBS washes, DAB detection was performed. Next, sections were washed two times in PBS and were counterstained with hematoxylin. After washing and dehydration, sections were mounted by the entellan mount.

\subsection{Statistical Analysis}

Normal assumption was checked for the tests. In the test, the P value for the diameter was $>0.05$, so the data was analyzed using one-way ANOVA followed by Post hoc LSD test and was presented as the mean \pm SD. The P value for the Johnson criteria and the height of epithelium was $<0.05$, so the data was analyzed using Kruskal-Wallis H. P $<0.05$ was considered significant. Data was analyzed using the SPSS software (version 16. SPSS Inc. United States)

\section{Results}

Normal spermatogenesis was seen in the control group, but degenerative changes in the germ cells and loss of spermatogenesis were seen in busulfan treated mice. Melatonin injection followed by busulfan treatment could improve spermatogenesis.

\subsection{Histopathologic Changes}

\subsubsection{Evaluation of Spermatogenesis}

Control testis showed normal spermatogenesis. Busulfan treatment destroyed testis tissue, and the mean John- son score (mean rank =15.50) was decreased significantly in comparison to the control group (mean rank=45.50), $\mathrm{P}<0.001$. Melatonin treatment following busulfan injection could nearly repair spermatogenesis, so a significant increase in the score (mean rank $=45.50$ ) was observed compared to the busulfan group (mean rank $=15.50$ ), $\mathrm{P}$ $<0.001$. Melatonin group showed a significant decrease in the Johnson score compared to the control group ( $\mathrm{P}$ $<0.05$ ) (Figure 2). As a result, melatonin reduced the damage of germinal cells due to busulfan treatment, but could not repair it completely.

\subsubsection{Seminiferous Tubule Morphometry}

In the current study, busulfan disrupted the seminiferous tubules structure, and the germinal cells were degenerated, except for small cells attached to the basement membrane. In addition, as seen in Figure 1 there are many vacuoles in the epithelium indicative of apoptosis in these cells. Melatonin injection could improve the damage (Figure 1). The mean diameter of the seminiferous tubules in the busulfan group (763.2 \pm 104.41$)$ indicated a significant decrease in comparison to the control (855.4 \pm 52.35$), \mathrm{P}<$ 0.01 , and melatonin groups (834.2 \pm 87.26 ), $\mathrm{P}<0.05$, (Table 1). Thickness of the seminiferous tubules in the busulfan group (mean rank =14.60) decreased significantly in comparison to the control (mean rank $=26.40$ ), $\mathrm{P}<0.01$, and melatonin groups (mean rank $=26.05$ ), $\mathrm{P}<0.01$. Also the melatonin group (mean rank $=25.42$ ) showed a significant reduction in epithelium height compared to the control group (mean rank=35.58), $\mathrm{P}<0.05$ (Figure 2 ).

Figure 1. H\&E Staining
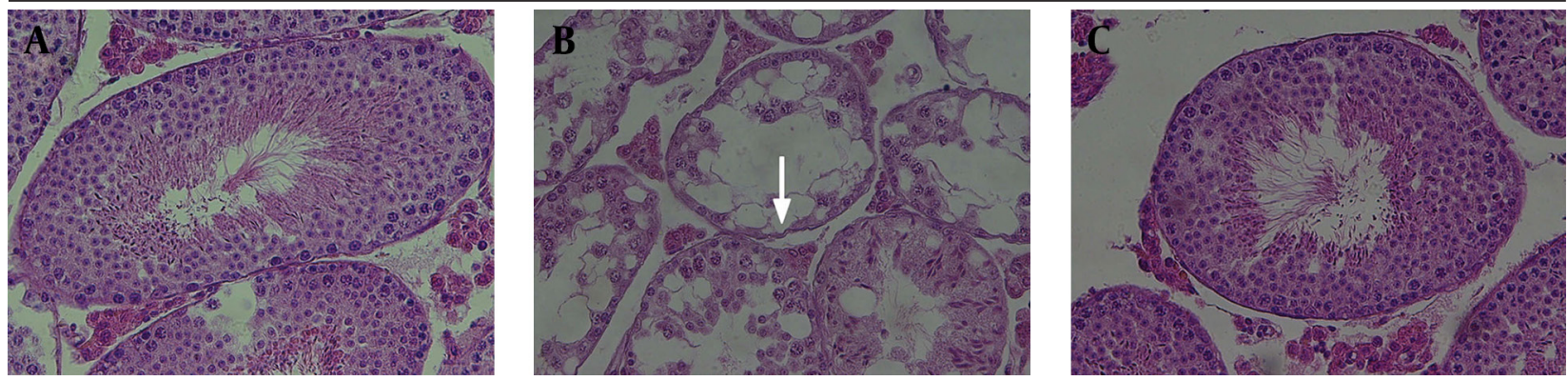

(A) The control group with normal spermatogenesis. (B) Section of testis of a busulfan treated mouse. Germinal layers are disorganized and there are many vacuoles in the tubules (arrow). (C) Cross section of mice testes, which received melatonin following busulfan treatment. GERM Cells have almost improved. H\&E Stained. Magnification: $\times 400$.

Table 1. Demographic Variables of Seminiferous Tubule Diameters

\begin{tabular}{llllll}
\hline Group & Mean & SD & SE & PValue & Confidence Interval (Lower, Upper Bound) \\
\hline Control & 855.40 & 52.35 & 11.70 & $0.003^{\mathrm{a}}, 0.708^{\mathrm{a}}$ & $(830.89,879.90)$ \\
Busulfan & 763.25 & 104.41 & 23.37 & $0.026,0.026^{\mathrm{a}}$ & $(714.38,812.11)$ \\
Melatonin & 834.25 & 87.26 & 19.51 & $0.708,0.026^{\mathrm{a}}$ & $(793.40,875.09)$ \\
Total & 817.63 & 91.79 & 11.85 & - & $(793.92,841.34)$ \\
\hline
\end{tabular}

${ }^{a}$ symbols indicate comparison to melatonin and busulfan, respectively. No symbol indicates the control group. 


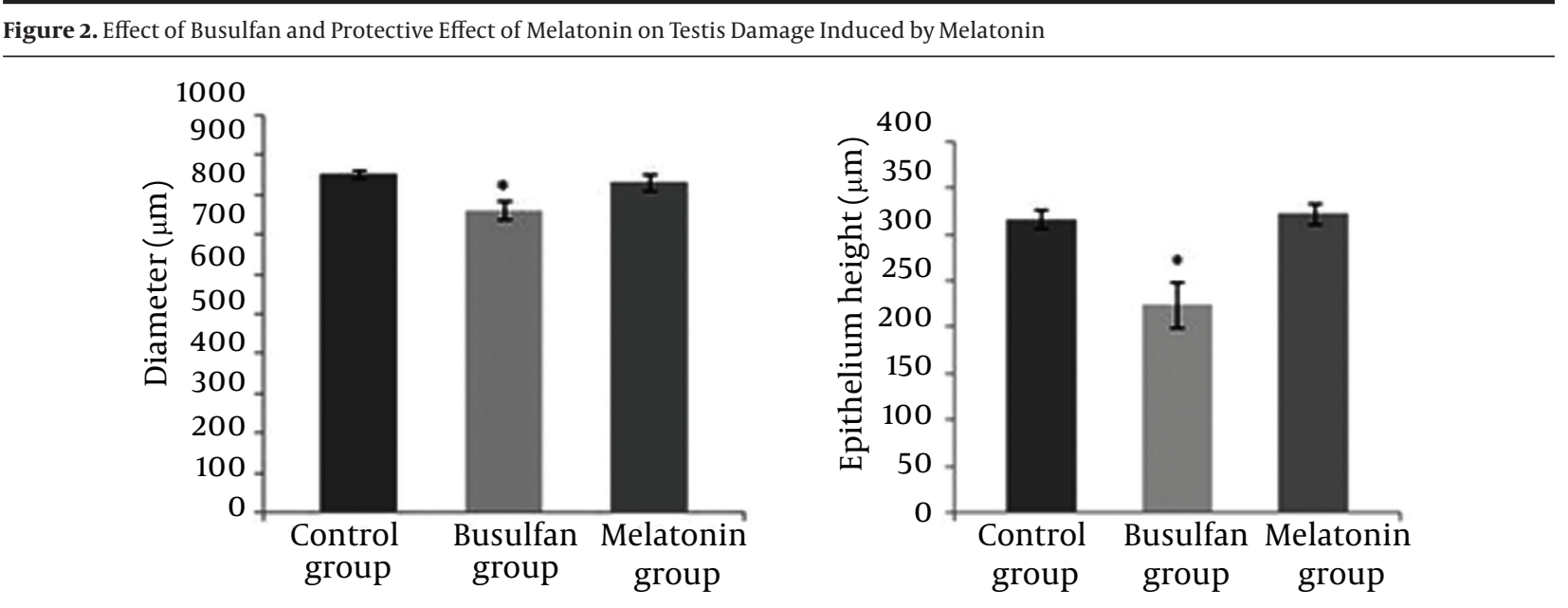

Busulfan reduced diameter and thickness of the seminiferous tubules, significantly. Melatonin could significantly repair the injury caused by busulfan. Values are shown as mean \pm SD
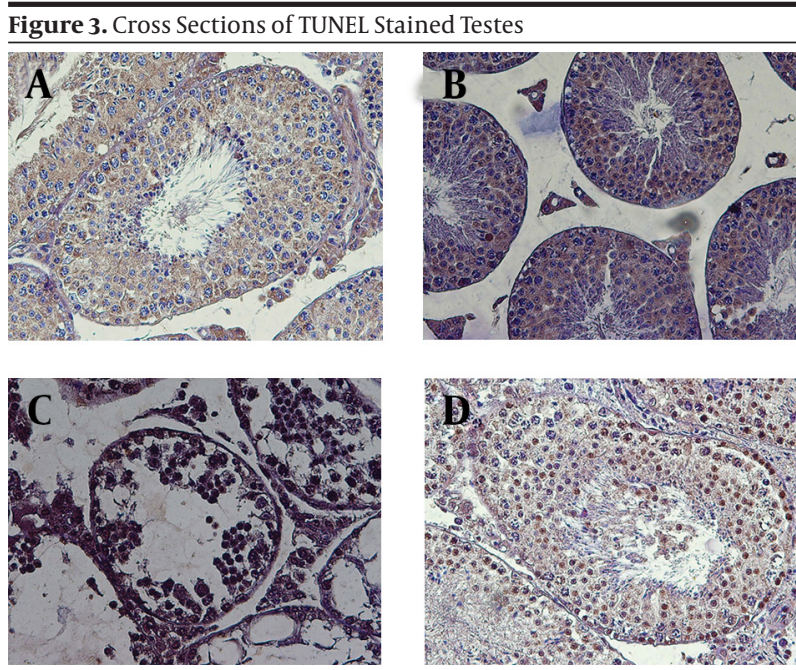

A; Negative control (all nucleuses are blue), B; Control group, which did not receive any drug. There are a few TUNEL positive cells. C; The group, which received busulfan. Most cells were TUNEL Positive. D; Melatonin was injected to mice after receiving busulfan. There were both apoptotic and normal cells. Note that apoptotic cells are indicated by brown nuclei. Magnification: $400 X$.

\subsubsection{Evaluation of Germ Cell Apoptosis}

TUNEL staining was performed to detect apoptotic cells in the testis tissue (Figure 3). There were only a few TUNEL-positive cells in the control group. Germ cells degeneration in the busulfan group was observed. In addition, TUNEL-positive germ cells also considerably increased in this group. Although melatonin reduced the destructive effects of busulfan on the testis, it could not significantly reduce the number of apoptotic germ cells in mice treated with busulfan.

\section{Discussion}

In this study, the germinal epithelium of male rats was destroyed by a single dose of busulfan, and it was shown that melatonin treatment could repair these defects. The fractionated dose of busulfan was determined based on a previous research (21). We observed the destructive action of busulfan on germ cells as shown in Figure1. Udagawa et al. indicated that busulfan treatment induces damage to spermatogenesis while recovery from such damage may be difficult (5). Many vacuoles were seen in the germinal epithelium in busulfan group. Vacuoles are considered as an apoptotic sign due to germ cell loss and detachment $(22,23)$. Apoptosis is a form of cell death, characterized by morphological and biochemical changes such as DNA fragmentation, formation of apoptotic bodies, and little inflammatory response (24).

Testicular apoptosis is found during normal spermatogenesis in mammals and is believed to be essential for the preservation of the correct ratio between sertoli cells and gametes (25). Germ cell apoptosis can also be significantly motivated by many pathological conditions such as heat stress, exposure to ionizing radiation, toxic substances, hormonal depletion, and loss of stem cell factor (SCF) (26). Increase in apoptotic rate in the busulfan group was confirmed by the TUNEL test compared to other groups. Apoptotic effects of busulfan were shown by earlier studies to cause a loss of A1 spermatogonia and primary spermatocytes (16).

Many efforts have been made to improve fertility after cytotoxic therapy; nearly all focused on hormonal treatment (27). Melatonin repaired the testis tissue in this study, and spermatogenesis was resumed after melatonin therapy as seen in Figure 1. Melatonin is synthesized by the pineal gland and is assumed to have free radical scavenger properties (11). Numerous studies have demonstrated the protective action of melatonin and their metabolites against oxidative and nitrosative changes of DNA $(11,28,29)$. These modifications are due to many en- 
dogenous and exogenous free radical producing processes (11). Melatonin was found to be a protectant against streptozotocin toxicity in diabetic rats (30) and after radiotherapy (31). In this research, although melatonin could reduce the rate of apoptosis in the busulfan group, many apoptotic cells were present after treatment. In agreement with our research, Hemadi et al. dedicated that melatonin can significantly reduce apoptosis, while inducing proliferation of the cells (14). Similarly Mohamad Ghasemi et al. reported reduced apoptosis following melatonin reception (17). These results are probably due to antioxidant properties of melatonin.

Evaluation of antioxidative status in the presence of melatonin is necessary to prove it. Considering that the germinal cells have receptors for melatonin, direct impact on the cells cannot be ruled out. On the contrary, Chen et al. (32) showed that melatonin induces apoptosis in germ cells of male mouse. In this case and some other studies such as that of Succu, melatonin was introduced as a time and dose dependent hormone (33). It has been shown that the prevalence of spermatogonial and spermatid apoptosis in Chinese men is more than Caucasian men (24). It appears that racial differences in the inherent sensitivity of germ cells to programmed cell death can be a factor for this difference. However, the mechanisms by which melatonin effect germ cells remain unknown. Finally, this study showed that melatonin improves the cytotoxic effect of busulfan on germ cells. Thus, it may be used for the protection of germinal layer defects caused by radiotherapy or chemotherapy in human. Obviously, more research is required to generalize these results to humans.

\section{Acknowledgements}

This study was part of a PhD thesis for Mehri Mirhoseini approved by the Physiology Research Center. Special thanks to Ahvaz Jundishapur University of Medical Sciences for their financial support.

\section{Funding Support}

This study was supported in part as an approved project by the Physiology Research Center of Ahvaz Jundishapur University of Medical Sciences. The funding organizations had no role in the design and conduct of the study, collection, management, and analysis of the data, or preparation, review, and approval of the manuscript. It had prepared animals for this research.

\section{Financial Disclosure}

The authors had no financial interests related to the materials in the manuscript.

\section{Authors' Contributions}

Study concept and design: Dr. Saki, Dr. Hemadi, Mirho- seini. Analysis and interpretation of data: Dr. Saki, Dr. Mohamadi Asl, Mirhoseini. Drafting the manuscript: Dr. Khodadadi, Mirhoseini. Critical revision of the manuscript for important intellectual content: Dr. Saki and Dr. Hemadi, Mirhoseini. Protocol development: Dr. Khodadadi, Mirhoseini. Statistical analysis: Mirhoseini.

\section{References}

1. Wyns C, Curaba M, Vanabelle B, Van Langendonckt A, Donnez J. Options for fertility preservation in prepubertal boys. Hum Reprod Update. 2010;16(3):312-28.

2. Mackie EJ, Radford M, Shalet SM. Gonadal function following chemotherapy for childhood Hodgkin's disease. Med Pediatr Oncol. 1996;27(2):74-8.

3. Kamischke A, Jurgens H, Hertle L, Berdel WE, Nieschlag E. Cryopreservation of sperm from adolescents and adults with malignancies. J Androl. 2004;25(4):586-92.

4. Wyns C, Curaba M, Martinez-Madrid B, Van Langendonckt A Francois-Xavier W, Donnez J. Spermatogonial survival after cryopreservation and short-term orthotopic immature human cryptorchid testicular tissue grafting to immunodeficient mice. Hum Reprod. 2007;22(6):1603-11.

5. Udagawa K, Ogawa T, Watanabe T, Yumura Y, Takeda M, Hosaka M. GnRH analog, leuprorelin acetate, promotes regeneration of rat spermatogenesis after severe chemical damage. Int J Urol. 2001;8(11):615-22.

6. Albrecht M, Tackmann W, Pribilla W. [Aplastic syndrome in myleran overdose]. Med Klin.1971;66(4):126-30.

7. Molenaar R, de Rooij DG, Rommerts FF, Reuvers PJ, van der Molen HJ. Specific destruction of Leydig cells in mature rats after in vivo administration of ethane dimethyl sulfonate. Biol Reprod. 1985;33(5):1213-22.

8. Bollag W. The effect of myleran on rat gonads. Experientia. 1953;9:268.

9. Shiraishi A, Sakumi K, Sekiguchi M. Increased susceptibility to chemotherapeutic alkylating agents of mice deficient in DNA repair methyltransferase. Carcinogenesis. 2000;21(10):1879-83.

10. Simone CB 2nd, Simone NL, Simone V, Simone CB. Antioxidants and other nutrients do not interfere with chemotherapy or radiation therapy and can increase kill and increase survival, part 1. Altern Ther Health Med. 2007;13(1):22-8.

11. Tan DX, Chen LD, Poeggeler B, Manchester LC, Reiter RJ. Melatonin: a potent, endogenous hydroxyl radical scavenger. Endocr J. 1993;1(4):57-60.

12. Dubocovich ML. Pharmacology and function of melatonin receptors. FASEB J.1988;2(12):2765-73.

13. Zisapel N. Melatonin receptors revisited. J Neural Transm. 1988;73(1):1-5.

14. Hemadi M, Zargar M, Sobhani A, Sobhani A. Assessment of morphological and functional changes in neonate vitrified testis grafts after host treatment with melatonin. Folia Morphol (Warsz). 2011;70(2):95-102.

15. Gholami MR, Saki G, Hemadi M, Khodadadi A, Mohammadi-As J. Supplementation vitrified-thawed media with melatonin do not protecting immature mouse testicular tissue from vitrifiedthawed induced injury. Asia J Anim Veterin Adva. 2012;7(10):940-9.

16. Choi YJ, Ok DW, Kwon DN, Chung JI, Kim HC, Yeo SM, et al. Murine male germ cell apoptosis induced by busulfan treatment correlates with loss of c-kit-expression in a Fas/FasL- and p53-independent manner. FEBS Lett. 2004;575(1-3):41-51.

17. Ghasemi FM, Faghani M, Khajehjahromi S, Bahadori M, Nasiri E E, Hemadi M. Effect of Melatonin on proliferative activity and apoptosis in spermatogenic cells in mouse under chemotherapy.J Reprod Contracept. 2010;21(2):79-94.

18. Ma YH, Hu JH, Zhou XG, Mei ZT, Fei J, Guo LH. Gamma-aminobutyric acid transporter (GAT1) overexpression in mouse affects the testicular morphology. Cell Res. 2000;10(1):59-69.

19. Orazizadeh M, Khorsandi LS, Hashemitabar M. Toxic effects of 
dexamethasone on mouse testicular germ cells. Andrologia. 2010;42(4):247-53.

20. Johnsen SG. Testicular biopsy score count-a method for registration of spermatogenesis in human testes: normal values and results in 335 hypogonadal males. Hormone Res Paediat. 1970;1(1):2-5.

21. Brinster CJ, Ryu BY, Avarbock MR, Karagenc L, Brinster RL, Orwig KE. Restoration of fertility by germ cell transplantation requires effective recipient preparation. Biol Reprod. 2003;69(2):412-20.

22. Sawada H, Esaki M. Electron microscopic observation of 137Csirradiated rat testis: production of basal laminae for germ cells, despite their absence. J Electron Microsc (Tokyo). 2003;52(4):391-7.

23. Sawhney P, Giammona CJ, Meistrich ML, Richburg JH. Cisplatininduced long-term failure of spermatogenesis in adult $\mathrm{C} 57 / \mathrm{Bl} / 6 \mathrm{~J}$ mice. J Androl. 2005;26(1):136-45.

24. Hikim APS, Swerdloff RS. Hormonal and genetic control of germ cell apoptosis in the testis. Reviews of Reproduction. 1999;4(1):38-47.

25. Lee J, Richburg JH, Younkin SC, Boekelheide K. The Fas system is a key regulator of germ cell apoptosis in the testis. Endocrinology. 1997;138(5):2081-8.

26. Ohta H, Aizawa S, Nishimune Y. Functional analysis of the p53 gene in apoptosis induced by heat stress or loss of stem cell factor signaling in mouse male germ cells. Biol Reprod.
2003;68(6):2249-54.

27. Meistrich ML, Wilson G, Huhtaniemi I. Hormonal treatment after cytotoxic therapy stimulates recovery of spermatogenesis. Cancer Res. 1999;59(15):3557-60.

28. Hardeland R, Tan DX, Reiter RJ. Kynuramines, metabolites of melatonin and other indoles: the resurrection of an almost forgotten class of biogenic amines. J Pineal Res. 2009;47(2):109-26.

29. Laothong U, Pinlaor P, Hiraku Y, Boonsiri P, Prakobwong S, Khoontawad J, et al. Protective effect of melatonin against Opisthorchis viverrini-induced oxidative and nitrosative DNA damage and liver injury in hamsters. J Pineal Res. 2010;49(3):271-82.

30. Guneli E, Tugyan K, Ozturk H, Gumustekin M, Cilaker S, Uysal N. Effect of melatonin on testicular damage in streptozotocininduced diabetes rats. Eur Surg Res. 2008;40(4):354-60.

31. Take G, Erdogan D, Helvacioglu F, Goktas G, Ozbey G, Uluoglu C, et al. Effect of melatonin and time of administration on irradiation-induced damage to rat testes. Braz J Med Biol Res. 2009;42(7):621-8.

32. Chen G. [Effect of melatonin on apoptosis of spermatogenic cells and expression of n NOS in testis of mice].J Anta. 2004;27(4):3835.

33. Succu S, Berlinguer F, Pasciu V, Satta V, Leoni GG, Naitana S. Melatonin protects ram spermatozoa from cryopreservation injuries in a dose-dependent manner.J Pineal Res. 2011;50(3):310-8 